\title{
Media, Biotechnology, and Trust: What Drives Citizens to Support Biotechnology
}

\author{
Zuoming Wang \\ Correspondence: Zuoming Wang, Department of Communication Studies, College of Liberal Arts \& Social Sciences, \\ University of North Texas, 1155 Union Circle \#305268, Denton, TX, USA 76203-5268, USA.
}

Received: November 20, 2017

Accepted: November 30, 2017

Online Published: November 30, 2017

doi:10.11114/smc.v5i2.2803

URL: https://doi.org/10.11114/smc.v5i2.2803

\begin{abstract}
This paper explores the relationship among ideology, media science news use, and three different types of trust (trust in government, trust in science, and trust in regulative institute), as well as their direct and indirect impact on the support of agricultural biotechnology and the support of regulation on genetic modified organisms (GMO). A structural model showed that the public are slightly leaning toward the use of biotechnology; meanwhile they overwhelmingly request the control on GMO. Level of education directly promoted all three types of trusts and had a positive effect on the support of biotechnology and the support of regulation on GMO. Media science news use has both a direct and an indirect positive effect (through trust in scientific process) on support of biotechnology. Among the three different types of trusts, trust on government did not have significant effects on public attitude toward biotechnology. Trust in regulative institutes, associated with more control on GMO, decreased the public's support of biotechnology. Meanwhile, trust in scientific process directly bolstered public's support of biotechnology.
\end{abstract}

Keywords: biotechnology, media, trust in government, trust in regulative institutes, trust in scientific process, genetic modified food

\section{Introduction}

\subsection{Biotechnology and GMO}

The introduction of biotechnology for food production and crop modification is a complex issue that goes beyond the scope of technology. With a moral component, as well as social and political implications, it has generated varying public responses across countries. The intensive debates have occurred not only on either side of the Atlantic: the United States (e.g., Nisbet \& Lewenstein, 2001; Priest, 2001; Shanahan, Scheufele, \& Lee, 2001), but also in European countries (see Bauer 2002, and Liakopoulos, 2002, in U. K.; Bondadelli, Dahinden, \& Leonarz, 2002, in Switzerland; Gutteling, 2002, in Netherlands; Kohring \& Matthes, 2002, in Germany; Lujan \& Todt, 2002, in Spain; Torgersen, 2002, in Austria), even in Asia (Yamaguichi, Harris, \& Busch, 2003, in India) and South Africa (Pouris, 2003). Research indicates that the public shows ambivalence toward the distinct applications of the biotechnology, "pointing out the crucial differences between general moral evaluation and attitude toward specific products" (Lujan \& Todt, 2000, p.383).

\subsection{Present Study's Importance}

Numerous studies have been conducted to gauge the public attitude toward the use of agricultural biotechnology, as well as identifying factors that shape the public opinion on this particular issue. However, the willingness for the public to be involved in the decision making process related to biotechnology issue is most often assumed rather than tested empirically (Brossard \& Shanahan, 2003). Therefore, what is the role of informed citizens in the decision making process? Does the knowledge on the biotechnology lead to more informed decision and more active public deliberation and participation? How does social trust relate to the democratic process in the public's policy making about biotechnology? Up to date, answers to these questions still remain unclear.

Our study examined these questions by studying the direct and indirect effects of ideology, media science news use, and different types of trusts (trust in government, trust in regulative institutes, and trust in scientific process) on the public's support of biotechnology, as well as to what extent, the public wants the control and regulation on the products of agricultural biotechnology (particularly GMO). Building upon previous studies, we proposed a model of public's attitudes on biotechnology that incorporates both content-specific media use about science and trust perceptions toward different actors. 


\subsection{Literature Review}

\subsubsection{Public's Perception about Biotechnology}

Brossard and Shanahan (2003) challenged the assumption that the public at large want to participate in the public deliberation about the use of agricultural biotechnology. They pointed out that citizens may "view agricultural biotechnology as more of a scientific than a political issue and thus do not believe public input is necessary" (p.292). In order to explore this argument empirically, they incorporated the public's authoritarian attitudes on biotechnology and tested "a model of decision making about biotechnology that links public's media use and authoritarian attitudes" (p.292). They further concluded that for such an important issue, the public may not want to be actively involved in the decision making process.

According to Brossard and Shanahan (2003), whether the public wants to be involved in the decision making process about the agricultural biotechnology to a large extent depends on how they interpret this issue. On one hand, if they believe that this is a purely scientific and technical issue, they may not consider that it's worthwhile generating and collecting the public input on this particular issue. Accordingly, they may give in their sayings and completely rely on the authorities, most often the scientists or the scientific institutes, to make the policy. On the other hand, if the use of agricultural biotechnology is considered as a social and political issue that affects people's daily life, the public may want to be more active in the decision making process. Since mass media are the important sources for people to obtain science information (Gerbner, Gross, \& Signorielli, 1981), questions about "Does mass media influence the public on how they look at the biotechnology?" and "What role the informed citizens play in the decision making process on biotechnology?" are salient on our agenda of biotechnology support research.

\subsubsection{The Role of Media on Public's Attitude of Biotechnology}

In modern society, mass media serve multiple roles in the opinion formation process. It not only provides the arena for information exchange and public deliberation (Habermas, 1979), sets the agenda for the public's attention (McCombs \& Shaw, 1993), but also directly influences the public opinion (Noelle-Neumann, 1984, 1993). Media are the primary source for the public to obtain knowledge about science and scientist (Brossard \& Shanahan, 2003). Content analysis of the media coverage reveals that science and scientists are frequently featured in mass media (Gerbner, Gross, \& Signorielli, 1981). Researchers found that media play an important role in informing the public about biotechnology (Gunter, Kinderlerer, \& Beyleveld, 1999), as well as shaping the public perception about science (Bauer, 2002; Besley \& Shanahan, 2005; Gutteling, 2002; Nisbet et al., 2002). Torgersen (2002) even suggest that "media campaigns can trigger technophobia among the uninformed public, and results in governments giving in to public pressure and abandoning sound science, and protectionism in agricultural policy that prevents free trade" (p.173).

All the above studies examined the general media use, but not content-specific information consumption about science and technology in the mass media. The extant literature suggests that media use in general influences the public's attitude toward the use of agricultural biotechnology; however, without looking into the content-specific news use on science, scholars could not conclude what exact role the scientific knowledge gained from the media plays in the public's attitude formation on the issue of biotechnology. In this sense, our study examined the media science news use and linked it with the public's trust in scientific process to explore the joint effects they produced on the public attitudes about biotechnology.

Prior research shows that overall a positive image of science and scientists is presented in the news and scientists are often portrayed as knowledgeable and trustworthy figures (Hornig, 1990; Nelkin, 1990). Moreover, the greater scientific knowledge is associated with less biased risk judgment (Brossard \& Shanahan, 2003). According to the cultivation perspective, the exposure to science news in the mass media increases the public's knowledge, familiarity, and favorability of science and scientists, and consequently boosts the trust in scientific process, and later translates into the public's support of biotechnology. Based on the above rationales, we raised the following hypotheses:

H1: Media science news use is positively related to trust in scientific process.

$\mathrm{H} 2$ : Media science news use is positively related to the support of the agricultural biotechnology use.

H3: Trust in scientific process is positively related to the support of the agricultural biotechnology use.

\subsubsection{Trust and Public Attitudes on Biotechnology}

The strong media's effect on public's biotechnology perception presented before was challenged by Priest (2001). She argued that the ability of media-based information to directly shape opinions on biotechnology is actually quite limited, and trust in institutions is more important than knowledge in predicting the support of specific applications of biotechnology. This argument was echoed by Bonfadelli, Dahinden and Leonarz (2002), who found that the public can be well informed but skeptical about the use of biotechnology. More knowledge on biotechnology does not translate into greater acceptance and higher support for its applications. 
Claiming that "education about the science... may nevertheless be largely irrelevant to attitude formation" (p.752), Priest and her colleagues (2003) proposed "trust gap" variables (defined as numerical differences between trust in specific pairs of actors) and found that "trust gap" did predict national levels of acceptance for biotechnology applications. On one hand, Priest's argument directs the attention to the important role of different types of trust in the public's attitude on biotechnology; on the other hand, her argument may be oversimplistic and tend to underestimate the media effect. Priest, et al., (2003) regard individual as rational actor making rational choices among which institutions to trust, and which ones are to be trusted more. Theoretically, this viewpoint shed new light on the research of the public's support of biotechnology. However, this rational choice argument is assumed rather than tested in their study. Moreover, numerous studies have showed that human beings are not always rational when confronted with complicated issues.

As discussed before, depending how the public interprets the issue of biotechnology, different types of trust needs to be examined. For a complex issue like biotechnology, its scientific proposition is intertwined with its moral, social and political implications. Brossard and Shanahan (2003) explored the role of the media in the information of authoritarian attitudes toward the democratic processes in science and found that media use was related to more authoritarian attitudes. Authoritarians are more likely to give up their independent thinking and refer to authorities for opinions. They tend to rely heavily on the authorities and reject the idea of democratic participation public deliberation (Altemeyer, 1996). The concept of trust is often related to the authoritarian attitudes. Depending on what the individuals perceive as the authorities, they may project trust toward different actors or agencies. Given the fact that government, regulative institutes, and scientists are usually portrayed as authorities in the mass media, authoritarians tend to have higher level of trust in those actors.

Brossard and Shanahan (2003) found that "authoritarians believe that authorities and experts should largely be trusted and that outcomes threatening the social order need to be controlled" (p.294). This suggests a potential relationship between trust in authorities and the desire to obtain control over potential risks. For an issue such as biotechnology, the public may want to keep an eye open for potential risks it could bring, therefore they may turn to authorities such as government or regulative institutes for more control. Based on these rationales, we predict that:

H4: Trust in government is positively related to the support of regulation on GMO.

H5: Trust in regulative institutes is positively related to the support of regulation on GMO.

Besides these hypotheses, we are also interested in the relationships among ideology and different types of trust. Thus, we raised the following two research questions:

RQ1: What's the relationship among trust in government, trust in regulative institutes, and trust in scientific process on the issue of biotechnology?

RQ2: What's the relationship between ideology and the three different types of trust?

\section{Method}

Data for our study came from a national telephone survey of 753 adults conducted in December 2013 (RDD sampling, response rate: $55 \%$ based on American Association of Public Opinion Research definition) by a Survey Research Institute located in a large university in northeast US. In order to ensure a true probability sample that would allow generalization to the larger population, multiple call-backs were made to all respondents over the course of one month in order to minimize non-response biases.

\subsection{Measures}

\subsubsection{Exogenous Variables}

Because age, gender and education have been shown to influence the attitudes toward science (Altemeyer, 1996; Schibeci, 1984; and Shanahan, 1995), we controlled all these demographic variables in the analysis. Our sample represented a good range of the population. The age of the participants ranged from 18 to 95 years old $(\mathrm{M}=50.08$ years, $\mathrm{SD}=17.16$ ). $54.8 \%$ of the sample were female with females coded 0 and males coded 1 . In terms of education, the number of years of education ranged from 3 to 22 years $(M=14.63$ years, $S D=2.95)$.

\subsubsection{Endogenous Variables}

Our measure of ideology $(M=8.24, S D=2.82)$ was an additive index of social and economical ideological leanings on seven-point scales $(r=.70)$ producing a 14-point scale of overall ideology. (The two questions are: The term "liberal" and "conservative" may mean different things to people, depending on the kind of issue one is considering. In terms of economic issues, would you say you are__. Now, thinking in terms of social issues and people's behavior, would you say you are . Answers ranging from 1: very liberal to 7 : very conservative). 
Media science news use $(M=10.36, S D=5.35)$ was broken down by medium. It was measured by combining two 10-point scales tapping people's attention to science and technology on both newspaper and television $(r=.79)$. (The two questions are: When you come across news about science and technology in the newspaper, how much attention do you pay to them? When you come across news about science or technology on TV, how much attention do you pay to them? Here one means little attention, and ten means very close attention)

Trust in government ( $M=4.55, S D=2.42)$ was a single item on 10 -point scale "I believe the Federal government looks out for my best interests." (1: strongly disagree, 10: strongly agree)

Trust in regulative institutes $(M=13.86, S D=4.33)$ was an additive index that consisted of two 10-point scale items ("Federal regulations will play a major role in preserving a clean and healthy environment" and "Federal regulations are absolutely necessary to protect American consumers." 1: strongly disagree, 10: strong agree) $(r=.44)$.

Trust in scientific process $(M=13.18, S D=4.21)$ was an additive index that consisted of two 10-point scale items ("Sound science must be our guide in choosing which foods are safe" and "Scientific tests can decide whether or not a food product is definitely safe." 1 : strongly disagree, 10 : strong agree $)(r=.28)$.

Support of biotech $(M=5.62, S D=2.79)$ was a single item on 10-point scale "Overall, would you say you oppose or support the use of biotechnology in agriculture and food production?" (1: strongly oppose 10: strongly support).

Support of regulation on $G M O(M=15.92, S D=3.98)$ was an additive index that consisted of two 10-point scale items ("There should be more control over genetically modified foods" and "All genetically modified foods must be labeled." 1: strongly disagree, 10: strong agree) $(r=.43)$.

\subsection{Generating the Model}

In order to establish the interconnections among all the above variables, we tested a theoretical model of structural relationships among these variables against our data by using LISREL (Jöreskog, 1993). LISREL allows for the simultaneous estimation of all parameters in a model. This model accounted not only for all the links from exogenous and endogenous variables to the dependent variable but also for the relationships among all exogenous and antecedent endogenous variables. Any given coefficient therefore represents the relationship between two variables, controlling for all other relationships and variables in the model. By treating endogenous variables as both independent and dependent variables, structural equation modeling allows for the estimation of direct and indirect effects. In this study, we followed the "model generating" approach (Jöreskog \& Sörbom, 1996) was followed. According to this approach, we developed an initial theoretical model and tested it with empirical data, then adjusted the model by freeing or fixing some of the paths based on the Lagrangian Multiplier (LM) test (Bollen, 1987) to optimize the model fit.

\section{Results}

In order to establish the interconnections between ideology, media science news use, trust in government, trust in regulative institutes, trust in scientific process, support of biotech, and support of regulation on GMO, we tested a theoretically-driven model of structural relationships among these variables against our data. This model accounts not only for all the links from exogenous and endogenous variables to the dependent variable but also for the relationships among all exogenous and endogenous variables.

The model fit the data exceptionally well with a Chi-Square of $22.08(\mathrm{df}=25, \mathrm{p}=.63, \mathrm{~N}=753)$. The ratio of Chi-Square and the $\mathrm{df}$ was .88, much less than the cutoff point 5. The Goodness-of-Fit Index (GFI) and the Adjusted-Goodness-of-Fit Index (AGFI) - controlling for multivariate non-normality - were both close to perfect with values of .99. The model explains $18 \%$ variance of ideology, $4 \%$ variance of media science news use, $8 \%$ variance of trust in government, $14 \%$ variance of trust in regulative institutes, $23 \%$ variance of trust in scientific process, $27 \%$ variance of support of biotech, and $42 \%$ variance of pro-regulation on GMO.

As is apparent from Figure 1, most of the predicted paths were statistically significant and in the hypothesized direction. All hypotheses were supported. The only exception is the expected link between trust in government and the support of regulation on GMO (H4). 


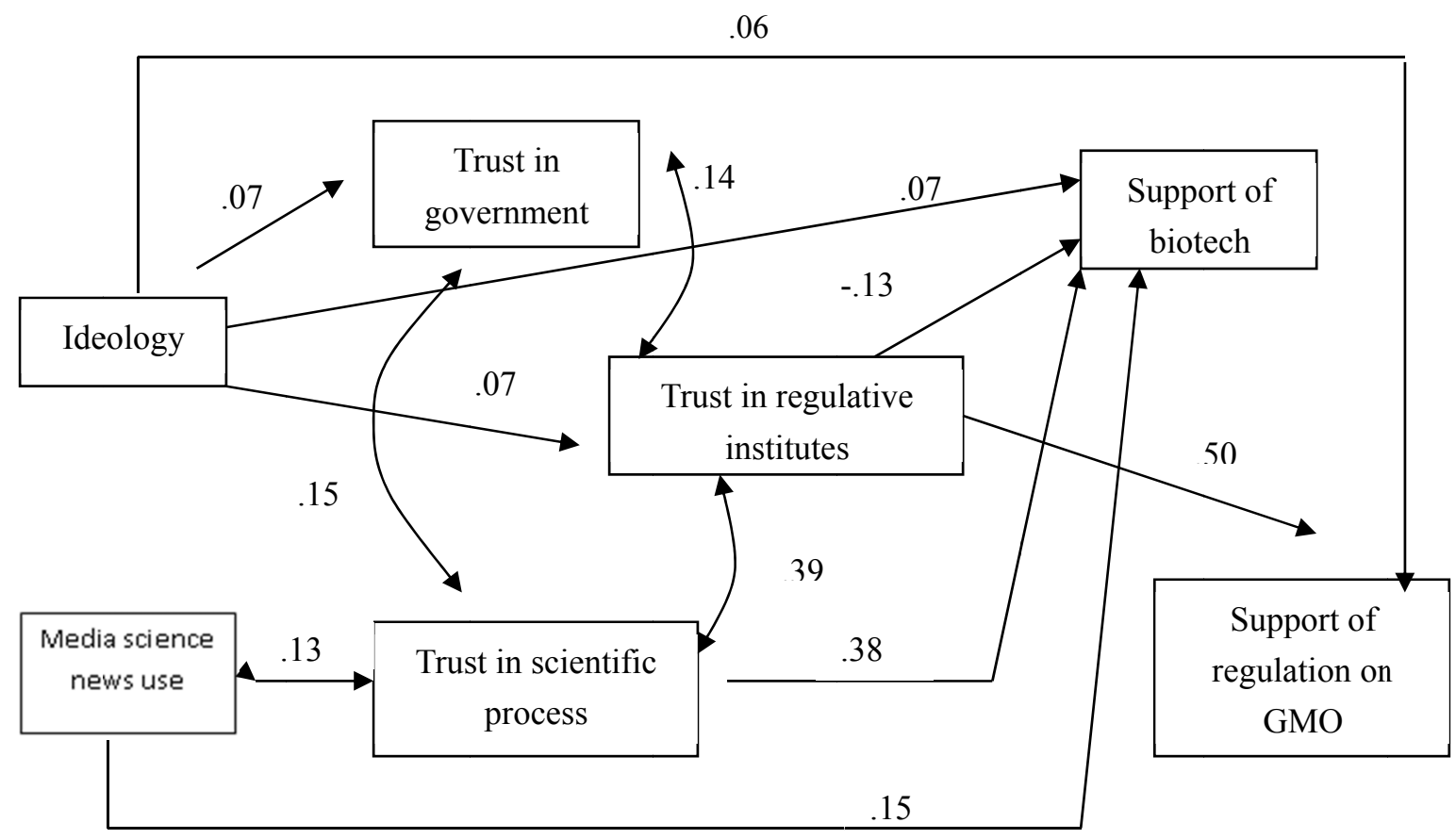

Figure 1. Structural model (demographic information controlled for). The full sample $(\mathrm{N}=753)$

As Table 1 shows that older respondents were more conservative $(\gamma=.14)$, which is consistent with prior research. Older respondents had higher level of trust in both regulative institutes $(\gamma=.10)$ and scientific process $(\gamma=.13)$. Generally speaking, they want more control and regulation on $\operatorname{GMO}(\gamma=.10)$. All the links between gender and other endogenous variables were not significant in the model, which suggests that with regard to the issue of agricultural biotechnology, men and women do not differ much on their attitudes. Highly educated respondents were more conservative $(\gamma=.29)$, and had higher level of media science news use $(\gamma=.20)$. The overall effect of education on trusts is important. Highly educated people tended to exhibit a higher level of trust on government $(\gamma=.13)$, on regulative institutes $(\gamma=.28)$, as well as on scientific process $(\gamma=.38)$. In general, highly educated respondents were more willing to support the use of biotechnology in agriculture and food production $(\gamma=.19)$, meanwhile they also desired more control and regulation on $\operatorname{GMO}(\gamma=.18)$.

Table 1. Influence of exogenous variables on endogenous variables in the model (the full sample: $N=753$ )

\begin{tabular}{llll}
\hline & Age & $\begin{array}{l}\text { Gender } \\
\text { (male) }\end{array}$ & Education \\
\hline \multirow{3}{*}{ Ideology } & .22 & --- & .29 \\
Media science news use & --- & --- & --- \\
& .22 & --- & .29 \\
Trust in government & --- & --- & .20 \\
& --- & --- & --- \\
Trust in regulative institutes & --- & --- & .20 \\
& --- & --- & .13 \\
Trust in scientific process & .02 & --- & .03 \\
& .02 & --- & .16 \\
Support of biotech & .10 & --- & .28 \\
& .02 & --- & .02 \\
Support of regulation on GMO & .12 & --- & .30 \\
& .13 & --- & .02 \\
& --- & --- & .40 \\
& .13 & --- & .19 \\
& --- & --- & .16 \\
& .05 & --- & .35 \\
& .05 & --- & .18 \\
& .10 & --- & .17 \\
\hline
\end{tabular}


Notes: (1) All coefficients are significant at $\mathrm{p}<.05$.

(2) Coefficients in the first row indicate direct effects, coefficients on the second row indicate indirect effects, and coefficients on the third row indicate total effects. If the sum of direct and indirect effects is not equal to the total effect size, this is due to nonsignificant indirect effects, which are not shown here.

Table 2 showed that ideology was directly related to trust in government $(\beta=.07)$ and the trust in regulative institutes $(\beta=.07)$. Conservative respondents trusted the government and regulative institutes more, which is consistent with the prior research. Meanwhile, the link between ideology and trust in scientific process was not significant, which suggests that conservative and liberal respondents did not differ much on their trust on science. Conservative respondents had higher level support of biotech $(\beta=.07)$, and they wanted more control and regulation on GMO as well $(\beta=.06)$. Respondents who attended to more media science news tended to have higher trust on scientific process $(\beta=.13)$ and more willing to support the biotech $(\beta=.15)$. Interestingly, trust in regulative institutes was positively related to more regulation on GMO $(\beta=.50)$, while it was negatively related to support of biotech $(\beta=-.13)$. Respondents who had higher level trust in scientific process supported the use of biotechnology in agriculture and food production more $(\beta=.38)$

Table 2. Relationships among endogenous variables (the full sample, $\mathrm{N}=753$ )

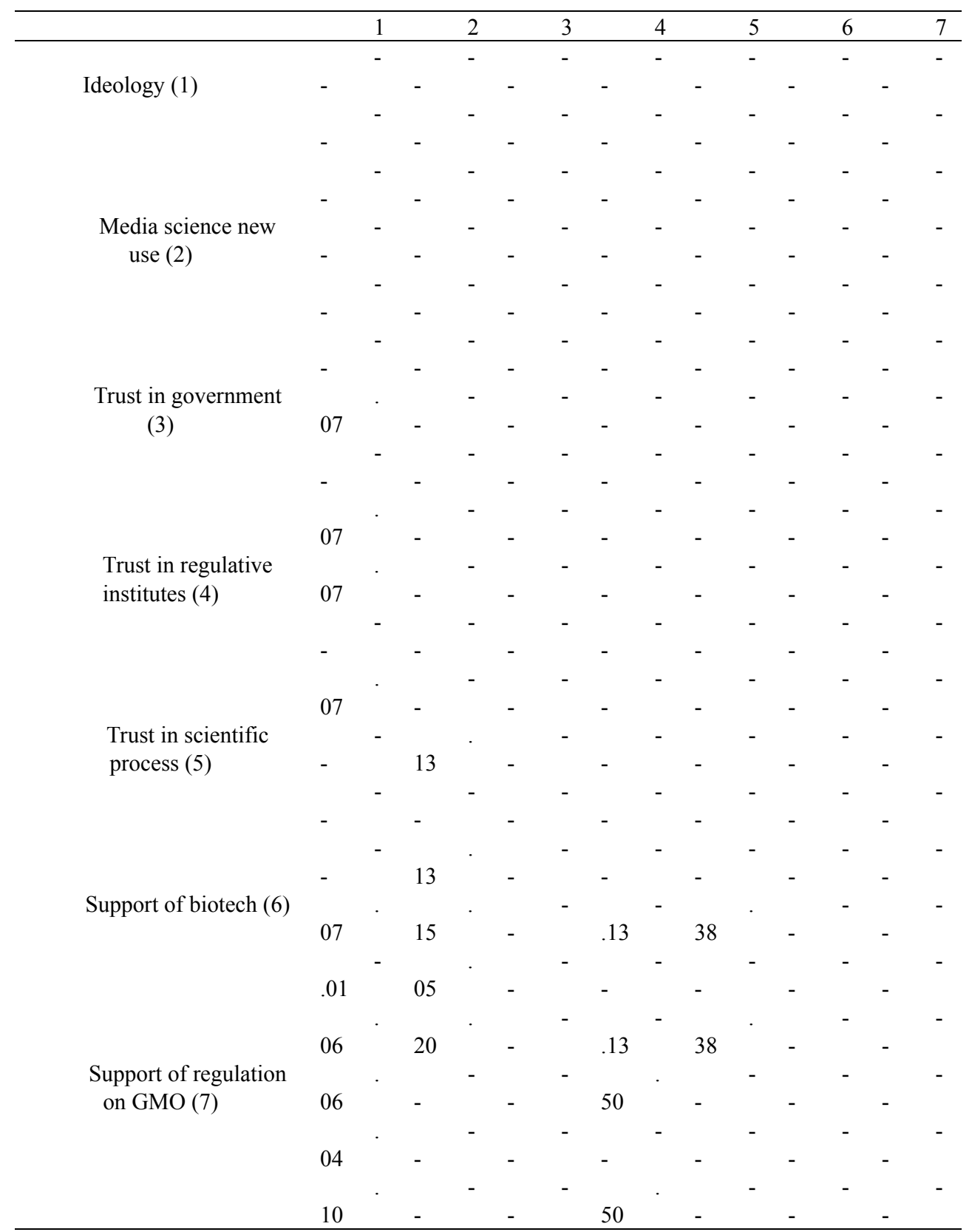

Notes: (1) All coefficients are significant at $\mathrm{p}<.05$. 
(2) Coefficients in the first row indicate direct effects, coefficients on the second row indicate indirect effects, and coefficients on the third row indicate total effects. If the sum of direct and indirect effects is not equal to the total effect size, this is due to nonsignificant indirect effects, which are not shown here.

In order to explore the potential differential effect of three different types of trust, we first presented respondents' scores on these three different trusts. Table 3 shows a large proportion of the respondents held low to moderate scores on their trust on government. While for trust in regulative institutes and trust in scientific process, majority of the respondents held moderate to high scores. This suggests that respondents allocated their trust differently to different actors.

Table 3. Percentage of respondents scoring low, moderate, and high on different types of trust

\begin{tabular}{lccc}
\hline & $\begin{array}{c}\text { Trust in } \\
\text { Government }\end{array}$ & $\begin{array}{c}\text { Trust in } \\
\text { Regulative } \\
\text { Institutes }\end{array}$ & $\begin{array}{c}\text { Trust in } \\
\text { Scientific } \\
\text { Process }\end{array}$ \\
\hline Low & $32.5 \%$ & $7.3 \%$ & $7.9 \%$ \\
Moderate & $54.7 \%$ & $44.6 \%$ & $42.7 \%$ \\
High & $12.9 \%$ & $48.1 \%$ & $49.4 \%$ \\
\hline
\end{tabular}

In order to explore the relationship among the three different types of trust, all links among them were freed, allowing them to be correlated to each other. LISREL automatically estimated the psi coefficient for those links. Results showed that they were all significant. The relationship between trust in government and trust in regulative institutes produced a psi coefficient of .14. Meanwhile, trust in scientific process was positively related to trust in government $(\psi=.15)$ and to trust in regulative institutes $(\psi=.39)$.

\section{Discussion}

This study examined two key questions. First, what is the role of the media science news use in promoting public support for biotechnology? Second, which type of trust is the essential factor for the public support for biotechnology? Our model tests the direct and indirect relationships among ideology, media science news use, three different types of trust (trust in government, trust in regulative institutes, and trust in scientific process), support of biotechnology, and the support of regulation on GMO. Although the effect of ideology on the public's support of biotechnology and the public's support of regulation on GMO is significant, this relationship is relatively weak, compared to media science news use and trusts.

Prior research shows inconsistent findings of the roles of the science knowledge and media science news use on the development of public opinion formation about biotechnology. On one hand, researchers have found that the public support of biotechnology is related to the knowledge about science (e.g., Brossard \& Shanahan, 2003; Wildavsky \& Dake, 1990), and the trust toward relevant governing bodies also affects the public perception (e.g., Yamaguchi, et al., 2003). On the other hand, another camp of scholars downplayed the role of the science literacy (e.g. Bonfadelli, et al., 2002; Priest, et al., 2003) and argued that public's reaction to biotechnology is a result of different trust and especially "trust gap" patterns rather than science education and knowledge (Priest, et al., 2003). Our study found that media science news use directly boosts the trust in scientific process, and increases the support of GMO both directly and indirectly (through trust in scientific process). This is consistent with previous studies which have detected the positive effect of media on public's perception on biotechnology (Bauer, 2002; Besley \& Shanahan, 2005; Gutteling, 2002; Nisbet et al., 2002; Torgersen, 2002). In other words, our study rejected the claims that science education and knowledge are irrelevant to public acceptance of biotechnology (Priest, et al., 2003). We have demonstrated that the effect of content-specific news consumption on science is not negligible in predicting public perception on biotechnology. We further argue that media science news use is intertwined with trust in scientific process and both factors jointly affect the public perception on biotechnology. Therefore, when studying the underlying mechanisms that drive the agricultural biotechnology support, we have to adopt a holistic perspective and examine both communication variables and trust perceptions, instead of overemphasizing or downplaying either of them.

Moreover, we detected differential effects of trusts on public support of biotechnology. Both trust in scientific process and trust in regulative institutes had a direct effect on public support on biotechnology, but in opposite directions. Trust in scientific process tended to increase the public support of biotechnology, while trust in regulative institutes tended to have a decreasing effect on it. An inspection of all factors that influence the public support of biotechnology revealed that trust in scientific process is the strongest and the most significant predictor. Brossard and Shanahan (2003) suggested that "agricultural biotechnology is considered too technological an issue to resonate with the general public" (p.301). Combining this explanation with our finding, we further suggest that given the sophisticated nature of the biotechnology, the public tends to give in their thoughts and turn to the authorities for rules and opinions on this issue, 
instead of independently developing their own ideas and actively engaging in the decision making process. For this particular case of biotechnology, the science and scientists stand out as the most trustworthy sources, which consequently drive the public's support of biotechnology. In comparison with the trust in scientific process, although the trust in regulative institutes also affects the public support of biotechnology in a negative way, the effect is much weaker. We also noticed that trust in government did not have a direct effect on the public support on biotechnology. A possible explanation is that the public does not consider the agricultural biotechnology as a political issue that the government needs to take the primary responsibility for. All these findings, to a large extent, supports the approach of examining different trusts and "trust gap" suggested by Priest, et al., (2003) when studying the public opinion on the issue of biotechnology. In other words, this study is consistent with Priest et al.'s (2003) argument that when confronted with the complicated issue of biotechnology, "a public (is) divided over who to believe rather than by degrees of mastery of scientific facts" (p.765).

With regard to the regulation on GMO, our study shows that only $2.5 \%$ of the respondents strongly disagree with a strict control on GMO. The majority (75.2\%) of the respondents demanded a strict control on GMO. Moreover, neither trust in government nor trust in scientific process plays a role in the public's support for regulation on GMO. Only trust in regulative institutes has a strong positive effect on it. This suggests that the public exhibits a strong need for keeping an eye open for the potential risks that agricultural biotechnology may cause. Meanwhile, the public regard regulative institutions as the primary actors for controlling these potential risks of GMO. The trust in regulative institutes and the public's need for a strong control over GMO is closely related.

By incorporating ideology, media science news use, and different types of trust in the model, this study enables us to understand more precisely how these factors help to foster the public attitudes on the biotechnology. Individuals' media science news use and their trust on different social actors play a central role in determining whether they will support the use of agricultural biotechnology, as well as to what extent the public wants the control and regulation on GMO. Our model shows the media science news use leads to higher trust in scientific process. The media science news use is also the strongest predictor for the public's support of biotechnology. Moreover, we found the impacts of different trusts on public attitude on biotechnology are sharply different. The trust in scientific process directly boosts the public's support of biotechnology. In contrast, the trust in regulative institutes hinders the public's acceptance of biotechnology. In sum, media science news use and the trust in scientific process are the two essential factors that influence the public support of biotechnology. Future research should examine the general media use and content-specific media information consumption, different types of trust, and the "trust gap" variables to better understand the public decision making process on the issue of biotechnology.

\section{References}

Altemeyer, R. (1996). The authoritarian specter. Cambridge, MA: Harvard University Press.

Bauer, M. W. (2002). Controversial medical and agri-food biotechnology: A cultivation analysis. Public Understanding of Science, 11, 93-111. https://doi.org/10.1088/0963-6625/11/2/301

Besley, J. C., \& Shanahan, J. (2005). Media attention and exposure in relation to support for agricultural biotechnology. Science Communication, 26, 1-21. https://doi.org/10.1177/1075547005275443

Bollen, K. A. (1987). Total, direct, and indirect effects in structural equation models. In C. C. Clogg (Ed.), Sociological methodology 1987, Volume 17 (pp. 37-69). San Francisco, CA: Jossey-Bass. https://doi.org/10.2307/271028

Bonfadelli, H., Dahinden, U., \& Leonarz, M. (2002). Biotechnology in Switzerland: High on the public agenda, but only moderate support. Public Understanding of Science, 11, 113-130. https://doi.org/10.1088/0963-6625/11/2/302

Brossard, D., \& Shanahan, J. (2003). Do citizens want to have their say? Media, agricultural biotechnology, and authoritarian views of democratic processes in science. Mass Communication and Society, 6, 291-312. https://doi.org/10.1207/S15327825MCS0603_4

Gerbner, G., Gross, L., \& Signorielli, N. (1981). Scientists on the TV screen. Culture and Society, 42, 51-54. https://doi.org/10.1007/BF02701349

Gunter, B., Kinderlerer, J., \& Beyleveld, D. (1999). The media and public understanding of biotechnology: A survey of scientists and journalists. Science Communication, 20, 373-394. https://doi.org/10.1177/1075547099020004002

Gutteling, M. J. (2002). Biotechnology in the Netherlands: Controversy or consensus? Public Understanding of Science, 11, 131-142. https://doi.org/10.1088/0963-6625/11/2/303

Habermas, J. (1979). Communication and the evolution of society. Boston: Beacon Press.

Hornig, S. (1990). Television's Nova and the construction of scientific truth. Critical Studies in Mass Communication, 7 , 11-23. https://doi.org/10.1080/15295039009360160 
Jöreskog, K. G. (1993). Testing structural equation models. In K. A. Bollen and J. S. Long (Eds.), Testing structural equation models (pp. 294-316). Newbury Park, CA: Sage.

Jöreskog, K. G., \& Sörbom, D. (1996). LISREL 8: User's reference guide. Chicago, IL: Scientific Software International.

Kohring, J., \& Matthes, J. (2002). The face(t)s of biotech in the nineties: How the German press framed modern biotechnology. Public Understanding of Science, 11, 143-154. https://doi.org/10.1088/0963-6625/11/2/304

Liakopoulos, M. (2002). Pandora's box or panacea? Using metaphors to create the public representation of biotechnology. Public Understanding of Science, 11, 5-32. https://doi.org/10.1088/0963-6625/11/1/301

Lujan, J. L., \& Todt, O. (2000). Perceptions, attitudes and ethical valuations: The ambivalence of the public image of biotechnology in Spain. Public Understanding of Science, 9, 383-392. https://doi.org/10.1088/0963-6625/9/4/303

McCombs, M. E., \& Shaw, D. L. (1993). The evolution of agenda-setting research-25 years in the marketplace of ideas. Journal of Communication, 43, 58-67. https://doi.org/10.1111/j.1460-2466.1993.tb01262.x

Nelkin, D. (1990). Selling science. Physics Today, 43, 41-46. https://doi.org/10.1063/1.881243

Nisbet, M. C., \& Lewenstein, B. V. (2002). Biotechnology and the American media: The policy process and the elite press, 1970 to 1999. Science Communication, 23, 359-391. https://doi.org/10.1177/107554700202300401

Nisbet, M. C., scheufele, D. A., Shanahan, J., Moy, P., Brossard, D., \& Lewenstein, B. V. (2002). Knowledge, reservations, or promise? Mass media and public perceptions of science. Communication Research, 29, 584-607. https://doi.org/10.1177/009365002236196

Noelle-Neumann, E. (1984, 1993). The spiral of silence: Public opinion-Our social skin. Chicago: University of Chicago Press.

Pouris, A. (2003). Assessing public support for biotechnology in South Africa. South African Journal of Science, 99, 513-516.

Priest, S. H. (2001). Misplaced faith: Communication variables as predicators of encourage for biotechnology development. Science Communication, 23, 97-110. https://doi.org/10.1177/1075547001023002002

Priest, S. H., Bonfadelli, H., \& Rusanen, M. (2003). The "trust gap" hypothesis: predicting support for biotechnology across national cultures as a function of trust in actors. Risk Analysis, 23, 751-766. https://doi.org/10.1111/1539-6924.00353

Schibeci, R. A. (1984). Attitudes to science: An update .Studies in Science Education, 11, 26-59. https://doi.org/10.1080/03057268408559913

Shanahan, J. (1995). Television viewing and adolescent authoritarianism. Journal of Adolescence, 18, 271-288. https://doi.org/10.1006/jado.1995.1019

Shanahan, J., Scheufele, D., \& Lee, E (2001). The polls - trends: Attitudes about agricultural biotechnology and genetically modified organisms. Public Opinion Quarterly, 65, 267-281. https://doi.org/10.1086/322201

Torgersen, J. (2002). Austria and the transatlantic agricultural biotechnology divide. Science Communication, 24, 173-183. https://doi.org/10.1177/107554702237843

Wildavsky, A., \& Dake, K. (1990). Theories of risk perception: Who fears what and why? Daedalus, 119, 41-50.

Yamaguchi, T., Harris, C. K., \& Busch, L. (2003). Agri-food biotechnology discourse in India. Science, Technology \& Society, 8, 47-72. https://doi.org/10.1177/097172180300800103

\section{Copyrights}

Copyright for this article is retained by the author(s), with first publication rights granted to the journal.

This is an open-access article distributed under the terms and conditions of the Creative Commons Attribution license which permits unrestricted use, distribution, and reproduction in any medium, provided the original work is properly cited. 